\title{
New measurements of magnetic fields of roAp stars with FORS 1 at the VLT`
}

\author{
S. Hubrig ${ }^{1}$, T. Szeifert ${ }^{1}$, M. Schöller ${ }^{1}$, G. Mathys ${ }^{1}$, and D. W. Kurtz ${ }^{2}$ \\ ${ }^{1}$ European Southern Observatory, Casilla 19001, Santiago 19, Chile \\ 2 Centre for Astrophysics, University of Central Lancashire, Preston PR1 2HE, UK
}

Received 27 May 2003 / Accepted 18 August 2003

\begin{abstract}
Magnetic fields play a key role in the pulsations of rapidly oscillating Ap (roAp) stars since they are a necessary ingredient of all pulsation excitation mechanisms proposed so far. This implies that the proper understanding of the seismological behaviour of the roAp stars requires knowledge of their magnetic fields. However, the magnetic fields of the roAp stars are not well studied. Here we present new results of measurements of the mean longitudinal field of 14 roAp stars obtained from low resolution spectropolarimetry with FORS 1 at the VLT.
\end{abstract}

Key words. stars: magnetic field - stars: oscillations - stars: chemically peculiar

\section{Introduction}

Helioseismology has proved a powerful tool to investigate important basic properties of the solar interior (Gough 2000). Likewise, asteroseismology has the potential to provide new insights into the physics of stellar interiors. Among the most promising objects that can be studied through this technique are the roAp stars. These are cool chemically peculiar stars which pulsate in high-overtone $(n \gg l)$, low-degree $(l \leq 3) p$-modes with periods from about 6 to $15 \mathrm{~min}$ and typical photometric $B$ amplitudes of a few mmag. There are 32 such stars currently known. Detailed reviews of the roAp stars have been published by Kurtz (1990), Matthews (1991), Martinez \& Kurtz (1995) and Kurtz (2003).

The pulsations of the roAp stars are strongly affected by their magnetic fields (Bigot et al. 2000; Cunha \& Gough 2000). Of particular importance is the recent work by Bigot $\&$ Dziembowski (2002). Their theory of the interaction of rotation, pulsation and the magnetic field suggests a new model, the improved oblique pulsator model, that is a significant departure from the standard oblique pulsator model for roAp stars. They suggest that the light variations are caused by a pulsation mode in which the stellar surface moves in a plane that is inclined to both the rotation and the magnetic axes of the star. The displacement vector describes an ellipse in that plane with the pulsation period, and the whole pattern rotates with the rotation of the oblique magnetic field.

Obviously, magnetic fields play a key role in the pulsations, which can only be properly interpreted if the strength and

\footnotetext{
Send offprint requests to: S. Hubrig, e-mail: shubrig@eso.org

* Based on observations obtained at the European Southern Observatory, Paranal, Chile (ESO programme No. 269.D-5044).
}

structure of these fields are known. Despite of the importance of magnetic fields for the proper understanding of pulsational properties of roAp stars, these fields have scarcely been studied until now. Among 32 roAp stars 20 definitely or very probably have a magnetic field (Mathys 2003), but of these 20, only three stars, HD 12098, HD 24712 and HD 83368, have been studied throughout their rotation periods (Lüftinger et al. 2003; Mathys \& Hubrig 1997). Here we present new results of measurements of the mean longitudinal field of 14 roAp stars obtained from low resolution spectropolarimetry with FORS 1 at the VLT.

\section{Observations and data reduction}

The observations reported here have been carried out between July and September 2002 at the European Southern Observatory with FORS 1 (FOcal Reducer low dispersion Spectrograph) mounted on the 8-m Melipal telescope of the VLT. This multi-mode instrument is equipped with polarization analyzing optics comprising super-achromatic half-wave and quarter-wave phase retarder plates, and a Wollaston prism with a beam divergence of $22^{\prime \prime}$ in standard resolution mode. For 13 stars we used the GRISM 600B in the wavelength range $3480-5890 \AA$ to cover all hydrogen Balmer lines from $\mathrm{H} \beta$ to the Balmer jump (Appenzeller et al. 1998). One star, HD 6532, has been observed with GRISM 600R in the region which includes $\mathrm{H} \alpha$ and $\mathrm{H} \beta$, from 4770 to $6900 \AA$ A. Both grisms have 600 grooves $\mathrm{mm}^{-1}$; with the narrowest available slit width of 0 '. 4 they give a spectral resolving power of $R \sim 2000$ and $R \sim 2900$, respectively.

Wavelength calibrations were taken during day time for the two different retarder waveplate setups $\left(\alpha=+45^{\circ}\right.$ and $\left.-45^{\circ}\right)$ that were used for the observations. Wavelength calibration was 
Table 1. Wavelength ranges around the hydrogen Balmer lines.

\begin{tabular}{cc}
\hline \hline Line $(\mathrm{s})$ & Wavelength range $[\AA]$ \\
\hline $\mathrm{H}_{16}-\mathrm{H}_{10}$ & $3701-3801$ \\
$\mathrm{H}_{9}$ & $3820.5-3852.5$ \\
$\mathrm{H}_{8}$ & $3870.2-3910.2$ \\
$\mathrm{H} \epsilon$ & $3941.2-4001.2$ \\
$\mathrm{H} \delta$ & $4082.9-4122.9$ \\
$\mathrm{H} \gamma$ & $4311.7-4371.7$ \\
$\mathrm{H} \beta$ & $4812.7-4912.7$ \\
$\mathrm{H} \alpha$ & $6512-6612$ \\
\hline
\end{tabular}

performed by associating with each individual science spectrum the calibration frame obtained with the similar orientation of the retarder waveplate. Ordinary and extraordinary beams were independently calibrated with the corresponding beams of the reference spectrum. As has been previously shown by Landstreet (1982), in the weak field regime, the mean longitudinal field can be derived from the difference between the circular polarizations observed in the red and blue wings of the hydrogen line profiles using the formula

$\frac{V}{I}=-\frac{g_{\mathrm{eff}} e \lambda^{2}}{4 \pi m_{\mathrm{e}} c^{2}} \frac{1}{I} \frac{\mathrm{d} I}{\mathrm{~d} \lambda}\left\langle\mathcal{B}_{z}\right\rangle$

where $V$ is the Stokes parameter which measures the circular polarization, $I$ is the intensity in the unpolarized spectrum, $g_{\text {eff }}$ is the effective Lande factor, $e$ is the electron charge, $\lambda$ is the wavelength, $m_{\mathrm{e}}$ the electron mass, $c$ the speed of light, $\mathrm{d} I / \mathrm{d} \lambda$ is the derivative of Stokes $I$, and $\left\langle\mathcal{B}_{z}\right\rangle$ is the mean longitudinal field. To minimize the cross-talk effect we executed the sequence $+45-45,-45+45,+45-45$ etc. and calculated the values $V / I$ using:

$\frac{V}{I}=\frac{1}{2}\left\{\left(\frac{f^{\mathrm{o}}-f^{\mathrm{e}}}{f^{\mathrm{o}}+f^{\mathrm{e}}}\right)_{\alpha=-45^{\circ}}-\left(\frac{f^{\mathrm{o}}-f^{\mathrm{e}}}{f^{\mathrm{o}}+f^{\mathrm{e}}}\right)_{\alpha=+45^{\circ}}\right\}$,

where $\alpha$ gives the position angle of the retarder waveplate and $f^{\mathrm{o}}$ and $f^{\mathrm{e}}$ are ordinary and extraordinary beams, respectively. Stokes $I$ values have been obtained from the sum of the ordinary and extraordinary beams. Table 1 lists the wavelength ranges of the hydrogen Balmer lines used in our calculations and for which we assumed a Landé factor $g_{\text {eff }}=1$. More details of the observing technique are given by Bagnulo et al. (2002) and Hubrig et al. (2003).

The errors of the measurements of the polarization have been determined from photon counting statistics and have been converted to errors of field measurements. For each roAp star we usually took 4 to 13 continuous series of 2 exposures with the retarder waveplate oriented at different angles. The spectropolarimetric capability of the FORS 1 instrument in combination with the large light collecting power of the VLT allowed us to achieve a $S / N$ ratio up to 2000 per pixel in the one-dimensional spectrum, as required to detect low polarization signatures produced in the spectral lines by longitudinal magnetic fields of the order of a few hundred gauss.

\section{Results}

The basic data of our sample of roAp stars are presented in Table 2. The columns are, in order, the HD number of the star, another identifier, the visual magnitude, the spectral type as it appears in the catalogue of Renson et al. (1991), the pulsation period, the heliocentric Julian date of mid-observation, number of series $N$ and the longitudinal magnetic field $\left\langle\mathcal{B}_{z}\right\rangle$ and its estimated uncertainty $\sigma_{z}$. The star HD 9563 shows the highest uncertainty of the magnetic field measurements $\left(\sigma_{z}=325 \mathrm{G}\right)$ because the exposure was taken in deteriorating weather conditions. Among the roAp stars studied, the rotation period is known only for the star HD $6532\left(P_{\text {rot }}=1\right.$ 1.94497; Kurtz et al. 1996).

In the following we give a brief overview of the previous knowledge of magnetic fields in the studied roAp stars. The most recent compilation of measurements of magnetic fields in roAp stars is given by Mathys (2003). Although the presence of a magnetic field is required by all the pulsation-driving mechanisms that have been proposed, knowledge of the magnetic fields of roAp stars is still very incomplete. Apart from HD 6532, all stars in our sample are poorly studied. For six of the stars presented here the spectropolarimetric magnetic field measurements are the first to have been made. We note here that the previous magnetic field data mentioned below are presented in greater detail in Mathys \& Hubrig (1997) and Mathys \& Hubrig (2003, in preparation).

\subsection{Notes on individual stars}

HD 6532: The 6.9-min pulsations in this star were discovered by Kurtz \& Kreidl (1985). This star is singly periodic with a rotationally modulated frequency quintuplet (Kurtz et al. 1996). It is also the only star in our sample for which a short rotation period $\left(P_{\text {rot }}=1\right.$. 94497 ) has been determined (see Kurtz et al. 1996 and references therein). The spectral lines show significant Doppler broadening which complicates the magnetic field determination using high-resolution spectropolarimetry (Mathys \& Hubrig 1997). An estimate of $v \sin i<47 \mathrm{~km} \mathrm{~s}^{-1}$ has been determined from CASPEC spectra obtained with the ESO $3.6 \mathrm{~m}$ spectrograph (Mathys \& Hubrig 2003, in preparation). The major advantage of using low-resolution spectropolarimetry with FORS 1 is that polarization can be detected in relatively fast rotators as we measure the field in the hydrogen Balmer lines. Our determination of the longitudinal field yields a nonzero value at $4.9 \sigma$ significance level.

HD 9289: The 10.5-min pulsations in this star were discovered by Kurtz (1993). A later study (Kurtz et al. 1994) found the star to be multi-periodic with alternating even and odd $\ell$-modes, similar to the well-studied roAp star HR 1217 (Kurtz et al. 2002). This star was observed only once with CASPEC (Mathys \& Hubrig 2003, in preparation), giving a nonzero longitudinal field of about $400 \mathrm{G}$. A longitudinal magnetic field of $(654 \pm 65) \mathrm{G}$ has been diagnosed from the FORS 1 spectra.

HD 12932: The 11.6-min pulsations in this star were discovered by Schneider \& Weiss (1990). This star is poorly studied, but appears to be singly periodic. A previous measurement with CASPEC (Mathys \& Hubrig 2003, in preparation) 
Table 2. Basic data of studied roAp stars and their magnetic fields measured with the FORS 1 instrument on the VLT UT3 (Melipal).

\begin{tabular}{|c|c|c|c|c|c|c|c|}
\hline $\mathrm{HD}$ & $\begin{array}{l}\text { Other } \\
\text { identifier }\end{array}$ & $V$ & Sp. type & $\begin{array}{r}P_{\text {puls }} \\
{[\mathrm{min}]}\end{array}$ & $\begin{array}{l}\text { HJD } \\
2452000+\end{array}$ & $N$ & $\begin{array}{c}\left\langle\mathcal{B}_{z}\right\rangle \\
{[\mathrm{G}]}\end{array}$ \\
\hline 6532 & $\mathrm{CD}-27^{\circ} 355$ & 8.4 & $\mathrm{~A} 3 \mathrm{SrCr}$ & 6.9 & 531.915 & 13 & $215 \pm 44$ \\
\hline 9289 & $\mathrm{BD}-11^{\circ} 286$ & 9.4 & $\mathrm{~A} 3 \mathrm{Sr} \mathrm{Eu}$ & 10.5 & 519.751 & 4 & $654 \pm 65$ \\
\hline 12932 & $\mathrm{BD}-19^{\circ} 384$ & 10.4 & $\mathrm{~A} 5 \mathrm{Sr} \mathrm{Eu}$ & 11.6 & 517.884 & 5 & $638 \pm 85$ \\
\hline 99563 & $\mathrm{BD}-08^{\circ} 3173$ & 8.2 & $\mathrm{~F} 0 \mathrm{Sr}$ & 11.2 & 494.483 & 5 & $-688 \pm 145$ \\
\hline 122970 & $\mathrm{BD}+06^{\circ} 2827$ & 8.3 & F0 & 11.1 & 494.500 & 5 & $216 \pm 56$ \\
\hline 161459 & $\mathrm{CD}-51^{\circ} 11145$ & 10.4 & $\mathrm{~A} 2 \mathrm{Eu} \mathrm{Sr} \mathrm{Cr}$ & 12.0 & 476.622 & 4 & $-1755 \pm 112$ \\
\hline 185256 & $\mathrm{CD}-30^{\circ} 17252$ & 9.9 & F0 Sr Eu & 10.2 & 476.676 & 4 & $-706 \pm 90$ \\
\hline 190290 & $\mathrm{CPD}-79^{\circ} 1062$ & 9.9 & A0 $\mathrm{Eu} \mathrm{Sr}$ & 7.3 & 494.561 & 5 & $3220 \pm 73$ \\
\hline 190290 & $\mathrm{CPD}-79^{\circ} 1062$ & 9.9 & $\mathrm{~A} 0 \mathrm{Eu} \mathrm{Sr}$ & 7.3 & 498.529 & 5 & $3250 \pm 111$ \\
\hline 193756 & CPD $-52^{\circ} 11681$ & 9.2 & A9 $\mathrm{Sr} \mathrm{Cr} \mathrm{Eu}$ & 13.0 & 498.575 & 10 & $-193 \pm 43$ \\
\hline 196470 & $\mathrm{BD}-18^{\circ} 5731$ & 9.8 & A2 Sr Eu & 10.8 & 476.732 & 4 & $1474 \pm 101$ \\
\hline 203932 & $\mathrm{CD}-30^{\circ} 18600$ & 8.8 & A5 $\mathrm{Sr} \mathrm{Eu}$ & 5.9 & 498.615 & 4 & $-267 \pm 72$ \\
\hline 213637 & $\mathrm{BD}-20^{\circ} 6447$ & 9.6 & $\mathrm{~F} 1 \mathrm{Eu} \mathrm{Sr} \mathrm{Cr}$ & 11.5 & 498.653 & 8 & $740 \pm 50$ \\
\hline 217522 & $\mathrm{CD}-45^{\circ} 14901$ & 7.5 & A5 $\mathrm{Sr} \mathrm{Eu} \mathrm{Cr}$ & 13.7 & 498.687 & 4 & $-725 \pm 88$ \\
\hline 218495 & CPD $-64^{\circ} 4322$ & 9.3 & $\mathrm{~A} 2 \mathrm{Eu} \mathrm{Sr}$ & 7.4 & 519.725 & 4 & $-912 \pm 71$ \\
\hline
\end{tabular}

revealed a longitudinal field of $400 \mathrm{G}$. From the FORS 1 spectra we derive $\left\langle\mathcal{B}_{z}\right\rangle=(638 \pm 85) \mathrm{G}$.

HD 99563: The 11.2-min pulsations in this star were discovered by Dorokhova \& Dorokhov (1998). The longitudinal magnetic field in this star at $4.7 \sigma$ level is reported here for the first time.

HD 122970: The 11.1-min pulsations in this star were discovered by Handler \& Paunzen (1999) and studied in greater detail in two multi-site campaigns by Handler et al. (2002) who found two modes of different degree, $\ell$, in both data sets, along with frequency and amplitude modulation. The presence of a longitudinal magnetic field in this star is reported here for the first time. Ryabchikova et al. (2000) determined a surface magnetic field of $2.3 \mathrm{kG}$ from the modelling of the rear-earth line profiles.

HD 161459: The 12.0-min pulsations in this star were discovered by Martinez \& Kauffmann (1991) and studied further by Martinez et al. (1991). The presence of a magnetic field in this star is reported here for the first time.

HD 185256: The 10.2-min pulsations in this star were discovered by Kurtz \& Martinez (1995). The presence of a magnetic field in this star is reported here for the first time.

HD 190290: The multi-periodic 7.3-min pulsations in this star were discovered by Martinez \& Kurtz (1990a) and studied further by Martinez et al. (1991). The presence of a magnetic field in this star is established here for the first time, via two measurements. This star shows the strongest longitudinal magnetic field (in absolute value) measured so far in any of the 32 known roAp stars. The two magnetic field measurements were separated by 4 days, but no significant variation was found between them.

HD 193756: The 13.0-min pulsations in this star were discovered by Martinez \& Kurtz (1990b). An attempt to detect the magnetic field in this star was made by Mathys \& Hubrig (1997), but no field was detected. The presence of a longitudinal magnetic field is provided by our present $4.5 \sigma$ measurement.

HD 196470: The 10.8-min pulsations in this star were discovered by Martinez et al. (1990). The presence of a magnetic field in this star is reported here for the first time.

HD 203932: The 5.9-min pulsations in this star were discovered by Kurtz (1984). This is one of the shortest periods known for the roAp stars; only HD 134214 has a shorter period at $5.7 \mathrm{~min}$. Further study by Martinez et al. (1990) indicated the presence of some transient frequencies. Two previous attempts to measure the magnetic field in this star (Mathys \& Hubrig 1997) yielded no detection. A longitudinal field of about $-150 \mathrm{G}$ was detected from a CASPEC spectrum by Mathys \& Hubrig (2003, in preparation). Our determination of the longitudinal field on the FORS1 spectra yields a detection at $3.7 \sigma$ level.

HD 213637: The 11.5-min pulsations in this star were discovered by Martinez et al. (1998). Resolved magnetically split lines have been observed in this star by Mathys (2003) and Kochukhov (2003).

HD 217522: The 13.7-min pulsations in this star were discovered by Kurtz (1983). Further observations by Kreidl et al. (1991) found another pulsation mode with a period of $8.3 \mathrm{~min}$ that was not present in the discovery data set, indicating transient modes, or strong amplitude modulation. The first magnetic observation of this star did not show any significant longitudinal magnetic field (Mathys \& Hubrig 1997). Here we report the detection of a longitudinal field from the FORS 1 spectra at the $8.2 \sigma$ level.

HD 218495: The 7.4-min pulsations in this star were discovered by Martinez \& Kurtz (1990b). Both previous 
determinations of the longitudinal field by Mathys \& Hubrig (1997) yielded nonzero values at a low level of significance. A longitudinal field at the $12.8 \sigma$ level has been diagnosed from the FORS 1 spectra.

\section{Discussion}

The presence of non-zero mean longitudinal fields in all roAp stars in our sample gives an indication that magnetic fields of a few hundred gauss up to a few kilogauss are widespread among roAp stars. Our results seem to hint that stronger magnetic fields tend to be found in hotter stars. Indeed, the stars of spectral type A0 and A2, HD 190290, HD 161459, HD 196470 and HD 218495 show the largest longitudinal fields. Hubrig et al. (2000a) have already reported about the existence of such a trend in their study of the evolutionary state of magnetic Ap stars. We should note that for roAp stars the effective temperatures derived from photometry are not in good agreement with the spectral classification (e.g., Kurtz 2002; North 2003, private communication). Because of the extremely anomalous energy distribution of these stars, the calibration of the photometric temperature indicators are frequently questioned. The inconsistencies can only be resolved by adopting effective temperatures inferred from detailed spectroscopic studies. In addition, because of the strong dependence of the longitudinal field on the rotational aspect, its usefulness to characterise actual field strength distributions is limited, but this can be overcome, at least in part, by repeated observations to sample various rotation phases, hence various aspects of the field.

Two related outstanding problems in our understanding of the nature of the roAp stars are the pulsation driving mechanism and the mode selection mechanism (see Shibahashi 2003 for discussion and references). The magnetic roAp stars pulsate only in high overtone modes while the non-magnetic $\delta$ Sct stars pulsate mostly in low overtone modes. Since these stars overlap in the H-R diagram, it is strongly suspected that the magnetic field plays a major role, whether directly or indirectly, in the driving and mode selection mechanisms. Except for HD 122970 and HD 217522, the stars in our sample do not have accurately determined Hipparcos parallaxes, hence their positions in the H-R diagram cannot be reliably determined. Nevertheless, in our recent study (Hubrig et al. 2000b) we were able to show that the domains of the roAp stars and the nonoscillating Ap (noAp) stars largely overlap in the H-R diagram. As this means that mass and internal structure differences between the roAp and noAp stars cannot be the only decisive factor in their respective evolution, the question arises whether another key factor plays a role in the excitation and mode selection mechanisms. The magnetic field is a likely candidate to be this factor. It is clearly necessary to understand the character of the surface magnetic fields in as much detail as possible.

These are the reasons why we are studying the magnetic field structure of the roAp stars. As mentioned by Mathys (2003) there is an unusually high frequency of occurrence of strong magnetic fields and of slow rotation among roAp stars. Seven roAp stars with magnetically resolved lines have already been detected since the discovery of pulsations more than 20 years ago. However, quite a number of roAp stars have rather weak or undetectable longitudinal fields and strong quadratic fields (Mathys \& Hubrig 1997). The determination of the quadratic field is based on the differential broadening that the field induces in unpolarized spectral lines having different magnetic sensitivities (for more details on the derivation of the quadratic magnetic field, see Mathys 1999). The rotational phase coverage achieved by the existing magnetic field data is insufficient and the structure of the magnetic fields in these roAp stars is presently unknown. Yet, from our results, it must be sufficiently tangled that it does not produce a strong observable polarization signature. Clearly, further systematic studies of magnetic fields in these stars should be conducted with a view to derive the geometrical structure of the fields. To understand better the origin of pulsations the derived models of magnetic fields must then be confronted with the pulsation properties of the roAp stars.

Acknowledgements. We are very grateful to S. Bagnulo for helpful discussions on data reduction. We would also like to thank P. North for the examination of the effective temperatures of the stars in our sample and the anonymous referee for the constructive comments which helped us to improve the paper.

\section{References}

Appenzeller, I., Fricke, K., Furtig, W., et al. 1998, The Messenger, 94, 1

Bagnulo, S., Szeifert, T., Wade, G. A., Landstreet, J. D., \& Mathys G. 2002, A\&A, 356, 218

Bigot, L., \& Dziembowski, W. A. 2002, A\&A, 391, 235

Bigot, L., Provost, J., Berthomieu, G., Dziembowski, W. A., \& Goode, P. 2000, A\&A, 356, 218

Cunha, M. S., \& Gough, D. 2000 MNRAS, 319, 102

Dorokhova, T. N., \& Dorokhov, N. I. 1998, Contributions of the Astronomical Observatory Skalnate Pleso, 27, 338

Gough, D. 2000, IAU Symp., 203, 529

Handler, G., \& Paunzen, E. 1999, A\&AS, 135, 57

Handler, G., Weiss, W. W., Paunzen, P., et al. 2002, MNRAS, 330, 153

Hubrig, S. North, P., \& Mathys, G. 2000a, ApJ, 539, 352

Hubrig, S., Kharchenko, N., Mathys, G., \& North, P. 2000b, A\&A, 355,1031

Hubrig, S., Bagnulo, S., Kurtz, D. W., et al. 2003, in International Conference On Magnetic Fields in O,B and A stars: Origin and Relation to Pulsation, Rotation and Mass Loss, ed. L. A. Balona, H. Henrichs, \& T. Medupe, ASP Conf. Ser., 216, in press

Kochukhov, O. 2003, A\&A, 404, 669

Kreidl, T. J., Kurtz, D. W., Bus, S. J., et al. 1991, MNRAS, 250, 477

Kurtz, D. W. 1983, MNRAS, 205, 3

Kurtz, D. W. 1984, MNRAS, 209, 841

Kurtz, D. W. 1990, ARA\&A, 28, 607

Kurtz, D. W. 1993, IBVS, 3966, 1

Kurtz, D. W. 2002, ASP Conf. Ser., 279, 351

Kurtz, D. W. 2003, Ap\&SS, 284, 29

Kurtz, D. W., \& Kreidl, T. J. 1985, MNRAS, 216, 987

Kurtz, D. W., \& Martinez, P. 1995, IBVS, 4209, 1

Kurtz, D. W., Martinez, P., \& Tripe, P. 1994, MNRAS, 271, 421

Kurtz, D. W., Marang, F., van Wyk, F., \& Roberts G. 1996, MNRAS, 280,1

Kurtz, D. W., Kawaler, S. D., Riddle, R. L., et al. 2002, MNRAS, 330, L57

Landstreet, J. D. 1982, ApJ, 258, 639 
Lüftinger, T., Kochukhov, O., Kuschnig, R., et al., 2003, in International Conference On Magnetic Fields in O,B and A stars: Origin and Relation to Pulsation, Rotation and Mass Loss, ed. L. A. Balona, H. Henrichs, \& T. Medupe, ASP Conf. Ser., 216, in press

Martinez, P., \& Kauffmann, G. 1991, IBVS, 3507, 1

Martinez, P., \& Kurtz, D. W. 1990a, IBVS, 3510, 1

Martinez, P., \& Kurtz, D. W. 1990b, IBVS, 3509, 1

Martinez, P., \& Kurtz, D. W. 1995, Ap\&SS, 230, 29

Martinez, P., Kurtz, D. W., Kauffmann, G., \& Jonson, A. C. 1990a, IBVS, 3506, 1

Martinez, P., Kurtz, D. W., \& Heller, C. H. 1990b, MNRAS, 246, 699

Martinez, P., Kurtz, D. W., \& Kauffmann, G. M. 1991, MNRAS, 250, 666

Martinez, P., Meintjes, P., Ratcliff, S. J., \& Engelbrecht, C. 1998, A\&A, 334, 606
Mathys, G. 1999, in Solar polarization, ed. K. N. Nagendra, \& J. O. Stenflo (Dordrecht: Kluwer), 489

Mathys, G. 2003, in International Conference On Magnetic Fields in $\mathrm{O}, \mathrm{B}$ and A stars: Origin and Relation to Pulsation, Rotation and Mass Loss, ed. L. A. Balona, H. Henrichs, \& T. Medupe, ASP Conf. Ser., 216, in press

Mathys, G., \& Hubrig, S. 1997, A\&AS, 124, 175

Matthews, J. M. 1991, PASP, 103, 5

Renson, R., Gerbaldi, M., \& Catalano, F. A. 1991, A\&A, 89, 429

Ryabchikova, T. A., Savanov, I. S., Hatzes, A. P., et al. 2000, A\&A, 357,981

Schneider, H., \& Weiss, W. W. 1990, IBVS, 3520, 1

Shibahashi, H. 2003, in International Conference On Magnetic Fields in O,B and A stars: Origin and Relation to Pulsation, Rotation and Mass Loss, ed. L. A. Balona, H. Henrichs, \& T. Medupe, ASP Conf. Ser., 216, in press 